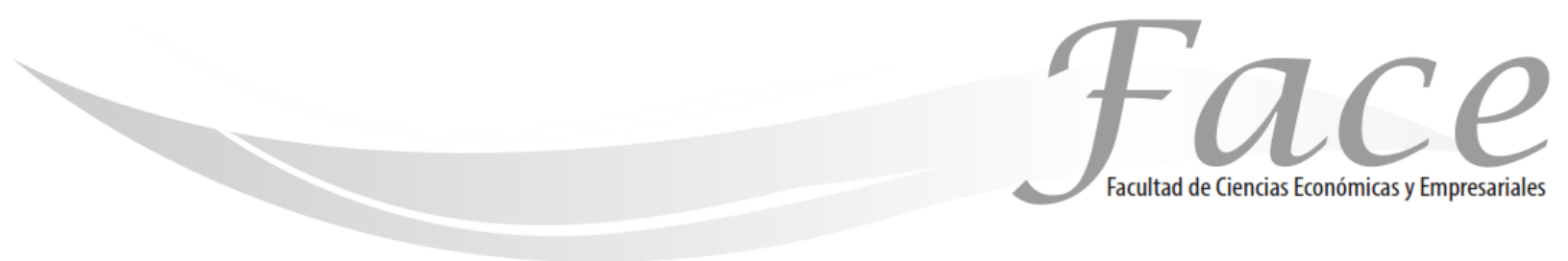

ISSN Impreso: 1794-9920 ISSN Electrónico: 2500-9338

Volumen $16-\mathrm{N}^{\circ} 2$

Año 2016

Págs. 110 - 116

\title{
PERCEPCIÓN DEL SECTOR SOLIDARIO DE PAMPLONA FRENTE A LAS NORMAS INTERNACIONALES NIIF*
}

\author{
Rina Esperanza Torres Vega ** \\ Enlace ORCID: http://orcid.org/0000-0003-2804-8391 \\ Laura Teresa Tuta Ramírez *** \\ Enlace ORCID: http://orcid.org/0000-0003-0938-1340 \\ Álvaro Parada Carvajal ${ }^{* * * *}$ \\ Enlace ORCID: http://orcid.org/0000-0003-4042-9891
}

Fecha de Recepción: 15 febrero 2016

Fecha de Aprobación: 28 mayo 2016

\section{Resumen:}

El auge de la globalización ha traído consigo importantes cambios en la economía de los negocios y en los procesos de internacionalización de las empresas, como es el caso de las normas internacionales de información financiera NIIF que afectan a todas las empresas, incluido el sector solidario; propiciando disminución de barreras culturales, de riesgos entre los asociados, entendimiento del lenguaje económico y contable en el que se desenvuelvan. Este estudio cuyo objetivo pretendió analizar la percepción de los representantes del sector solidario de Pamplona (N. de S.) frente a las normas internacionales NIIF, se adelantó desde una investigación descriptiva, con un método deductivo y enfoque cuantitativo que permitió mediante encuestas, analizar a 37 organizaciones del sector solidario de Pamplona. El sustento teórico de este estudio está basado en los contenidos de Casinelli (2008), Berrio (2015), Figueroa (2007), entre otros. Como resultado del estudio los gerentes del sector asintieron la importancia y la obligatoriedad legal de implementar las NIIF, asimismo, solicitaron asesoría pues la mayoría como Mi pymes no cuentan con recursos ni personal experto en el área, por lo que se les diseñó y entregó una cartilla didáctica.

Palabras Claves: Normas internacionales NIIF, Percepción empresarial, sector solidario.

* Contador Público, Universidad de Pamplona. rinaet@hotmail.com

* Administradora de Empresas, Ph D. Ciencias Gerenciales, Mg. Gerencia de Empresas-Mercadeo, Docente tiempo completo Universidad de Pamplona,

Investigador Junior Colciencias, Grupo de Investigación GICEE. laurat@unipamplona.edu.co

* Administrador de Empresas, Esp. Relaciones Industriales, docente de Tiempo Completo Universidad de Pamplona, grupo de investigación CEyCON.

alpaca@unipamplona.edu.co 


\title{
PERCEPTION OF THE SOLIDARITY SECTOR OF PAMPLONA AGAINST INTERNATIONAL STANDARDS IFRS
}

\begin{abstract}
The rise of globalization has brought significant changes in the economy of business and the internationalization of companies, such as the International Financial Reporting Standards IFRS that affecting all companies, including the solidarity sector; promoting reduction of cultural barriers, risk among partners, understanding of the economic and accounting language in which they unfold. This study aimed to analyze the perception of the representatives of the solidarity sector of Pamplona (N. S.) about against international standards IFRS, it was conducted by a descriptive research with a quantitative approach and deductive method, it allowed through surveys, the analyze 37 organizations of the solidarity sector of Pamplona. The theoretical basis of this study is based on the contents of Casinelli (2008), Berrio (2015), Figueroa (2007), among others. As a result of the study sector managers they assented the importance and legal obligation to implement IFRS also requested advice as most as MSMEs do not have resources or skilled personnel in the area, which were designed and delivered a primer teaching.
\end{abstract}

Keywords: International standards IFRS, Business Perception, solidarity sector.

\section{PERCEPÇÃO DO SECTOR DA SOLIDARIDADE DE PAMPLONA CONTRA NORMAS INTERNACIONAIS IFRS *}

\begin{abstract}
Resumo
O aumento da globalização trouxe consigo mudanças importantes na economia empresarial e nos processos de internacionalização das empresas, como no caso dos padrões de relatórios financeiros internacionais que afetam todas as empresas, inclusive o setor solidário; promovendo uma redução das barreiras culturais, riscos entre associados, compreensão da linguagem econômica e contábil em que se desenvolvem. Este estudo teve como objetivo analisar a percepção dos representantes do setor solidário de Pamplona (N. de S.) em relação aos padrões internacionais IFRS, foi avançado a partir de uma pesquisa descritiva, com método dedutivo e abordagem quantitativa que permitiu, através de pesquisas, analisar a 37 organizações do setor solidário de Pamplona. A base teórica deste estudo baseia-se nos conteúdos de Casinelli (2008), Berrio (2015), Figueroa (2007), entre outros. Como resultado do estudo, os gerentes do setor concordaram com a importância e obrigação legal de implementar as IFRS. Eles também solicitaram conselhos, uma vez que a maioria deles como MPMEs não tem recursos ou conhecimentos na área, então eles foram projetados e recebidos um primer didática.
\end{abstract}

Palavras-chave: Normas Internacionais IFRS, Percepção Empresarial, Setor de Solidariedade. 


\section{INTRODUCCIÓN:}

A nivel mundial las economías están hoy más dispuestas a aceptar la inversión extranjera buscando mayores oportunidades al traspasar las fronteras. Sin embargo, esta inversión representa un gran reto debido a que las empresas se enfrentan a circunstancias nuevas y en constante cambio. En este contexto, Colombia avanza en la legalización de tratados de libre comercio que permitan el acceso en doble vía a mercados extranjeros para incentivar el intercambio de conocimientos, transacciones económicas y el flujo de capital entre los países que firmen los tratados, por tal motivo es importante unificar el lenguaje contable que le permita a las empresas ser competitivas a nivel global. En consecuencia, el Congreso de la República (2009) expidió la Ley 1314, que regula los principios y normas de contabilidad e información financiera y de aseguramiento de información, donde se señala el procedimiento para su expedición y se determinan las entidades responsables de reglamentar $y$ de vigilar su cumplimiento.

Por consiguiente, es necesario analizar el estado de capacidades y posibilidades que presentan los sectores económicos, pues según informe del Departamento Administrativo Nacional de Estadística (DANE, 2016), el grado de informalidad que tiene Colombia en datos de noviembre de 2015 es del $47,1 \%$, cifra bastante elevada; es decir, no existe seguridad de cultura plena para la creación de empresa y las existentes en su mayoría carecen de compromiso de alta gerencia y planeación estratégica para competir en mercados internacionales.

Frente a este contexto, preocupa que ante el requisito de aplicación de la Ley de regulación de las normas internacionales de información financiera NIIF, que naturalmente acepta y adopta el país; para muchos empresarios aún sea desconocida la función jurídica, contable, financiera, su funcionalidad en unificación de un mismo lenguaje que permita al empresario aplicar una contabilidad común en todos sus aspectos, implicando reducción de costos, mejora de los canales de comunicación, confianza en procesos de internacionalización; en detrimento del acceso a mejores oportunidades de mercado y de implicaciones de fuertes sanciones.

En este sentido, es claro que la normativa, afecta a todas las empresas, incluyendo al sector solidario y que según Berrio (2015), en Colombia en el año 2014, éste participa con el 4,9\% del producto interno bruto, aproximadamente generando más de 15.200 millones de dólares anuales, más de 512 mil empleos formales y aproximadamente hay 6.500 cooperativas haciendo importantes aportes a la economía y la sociedad nacional.

Por consiguiente, siendo éste uno de los sectores que ejerce alto impacto en el desarrollo socio-económico de Pamplona, Norte de Santander, constituido por variedad de cooperativas y asociaciones cuyo incremento se acerca a un centenar, Cámara de Comercio de Pamplona (2016), surge el interés de adelantar esta investigación de tipo descriptivo, con un enfoque cuantitativo y mediante un método deductivo que parte de la amplitud de la norma para contactar a 37 empresarios del sector y abordarlos mediante encuesta, con el fin de analizar la percepción de los representantes del sector solidario de Pamplona ( $\mathrm{N}$. de S.) frente a las normas internacionales NIIF, identificar las condiciones legales y reglamentarias que presentan las empresas del sector para la adopción de las normas internacionales NIIF y poder proponer estrategias de acompañamiento a los empresarios del sector en la implementación de dichas Normas.

En esta línea, se procuró el sustento teórico tomando como referencia los postulados internacionales, la Ley 1314 y las bases teóricas de Casinelli (2008), Berrio (2015), Figueroa (2007), entre otros. Asimismo, soportados en el proceso metodológico, el análisis de datos mostró como resultados la descripción de la manifestación perceptiva por parte de los empresarios que dirigen las empresas, también la situación en que se encuentran a nivel legal, la capacidad y disposición frente a la implementación de las NIIF en el sector solidario de la ciudad.

\section{MARCO TEÓRICO:}

Fundamentación teórica del estudio

Se exponen apartes teóricos que llevan a reflexionar la importancia de analizar y comprender de manera oportuna la situación real respecto de la implementación de las NIIF en las empresas, especialmente si trata de pymes 0 de organizaciones del sector solidario dada la complejidad del proceso y el alcance totalitario de la Ley.

2.1 Posturas sobre el componente legal y reglamentario NIIF

En Colombia, la adopción de las normas internacionales de contabilidad, es un tema que se viene tratando hace ya algunos años, pero por algunas discrepancias relacionadas al fuerte cambio y la responsabilidad que deben enfrentar se ha estado aplazando.

Desde este contexto, el Congreso de la República (2009), promulga la Ley 1314 (Reglamentada por el Decreto Nacional 1851 de 2013 y por el Decreto Nacional 302 de 2015), donde regula los principios y normas de contabilidad e información financiera y de aseguramiento de información aceptados en Colombia, se señalan las autoridades competentes, el procedimiento para su expedición y se determinan las entidades responsables de vigilar su cumplimiento. Frente a la convergencia de las 
normas contables nacionales con las de orden internacional, establece que el Consejo Técnico de la Contaduría Pública es el único autorizado para redactar las normas de convergencia, y fija como plazo para el inicio de la convergencia desde el año 2010 hasta el 2014.

Estas NIIF asumen los principios de equidad, reciprocidad y conveniencia nacional, bajo el propósito de apoyar la internacionalización de las relaciones económicas, por consiguiente el Estado promueve la convergencia de tales normas de contabilidad, información financiera, el aseguramiento de la información en términos de estándares internacionales de aceptación mundial, con las mejores prácticas y con la rápida evolución de los negocios. Pretende visionar grandes oportunidades de crecimiento y evitar sufrir serias consecuencias.

En este sentido, lo reseñado por (Luna y Muñoz, 2011), deja entrever el cuidado en la adopción de las NIIF con el fin de que la armonización entre estas normas se haga de forma adecuada, sin perjudicar a los usuarios de la información contable (internos y externos), también el reto de los profesionales del ramo, cuyo esfuerzo en capacitaciones es urgente en la aplicación de las NIIF para desenvolverse apropiadamente en el nuevo ambiente de economías globalizadas. Las mencionadas autoras destacan que el Consejo Permanente para la Evaluación de las Normas sobre Contabilidad se ha mostrado pausado en cuanto a esa armonización de las normas internas con las NIIF, retrasando la incorporación de Colombia a diversos convenios o tratados internacionales que exigen cada día la preparación de estados financieros más rigurosos, con indicadores de alta calidad y transparencia, la revaloración conceptual como el de mantenimiento del capital y reconocimiento de los ingresos por el término genérico de desempeño financiero.

Por otra parte, Colombia como integrante de la Organización Mundial del Comercio (OMC) ha tomado consciencia de la globalización actual, razón por la cual el Congreso de la República (1999), al expedir la Ley 550 estableció en su artículo 63 llamado armonización de las normas contables con los usos y reglas internacionales que para efectos de garantizar la calidad, suficiencia y oportunidad de la información que se suministre a los asociados y a terceros, el Gobierno Nacional revisaría las normas actuales en materia de contabilidad, auditoría, revisoría fiscal y divulgación de información, con el objeto de ajustarlas a los parámetros internacionales y proponer al Congreso las modificaciones pertinentes.

Desde otra perspectiva, Casinelli (2008), planeta que la información financiera y en especial la denominada información contable, se ha convertido en el lenguaje de los negocios, es decir, aquel que utilizan las empresas para comunicarse con los establecimientos de crédito, accionistas, potenciales inversionistas, proveedores, clientes o hacienda pública.

\subsection{Postura normatividad de las NIIF a nivel internacional}

Según lo descrito por (Figueroa; 2007) las normas internacionales de contabilidad, también denominadas NIC, son un conjunto de postulados generales de Contabilidad, que tienen como fin estandarizar a nivel mundial las normas con las que cada país lleva la Contabilidad. Aclara: "Dichas normas, fueron creadas por el International Accounting Standards Committee, IASC, organismo que fue conformado en 1973 por profesionales de Contabilidad representantes de países como: Alemania, Australia, Canadá, Francia, Países Bajos, Japón, México, Reino Unido/Irlanda y Estados Unidos. (p,11). Así sustituyó al IASC en la emisión de normas contables; en 2001 fue creado el IASB, International Accounting Standards Board, siendo el único organismo profesional que se encarga de emitir las NIC-NIIF.

\subsection{Posturas NIIF con enfoque al sector solidario}

De acuerdo con el Consejo Técnico de la Contaduría Pública CTCP (2012), la economía solidaria a partir de los postulados de libre adhesión, retiro voluntario y capital variable e ilimitado, se convierte en un verdadero reto financiero, económico y normativo cuando se trata de efectuar la convergencia hacia normas internacionales de contabilidad (NIC) y Normas Internacionales de Reporte Financiero (NIIF). Debido a lo anterior se ha hecho necesario que las cooperativas determinen y definan el proceso a seguir conforme al tipo de normas que deban aplicar. A nivel normativo se establece a consideración la información al corte de diciembre de 2012, más la organización y el principio de autonomía administrativa. Igualmente se promulga para cada grupo el proceso de convergencia.

Además, Berrio (2015) en temas de organización y clasificación que abarca al sector solidario, expone que se propuso en el párrafo 48 que las normas 51 de contabilidad e información financiera y de aseguramiento de la información deben aplicarse de manera diferencial a tres grupos de preparadores de estados financieros: Grupo 1 (NIIF plenas), Grupo 2 (NIIF Pymes) y Grupo 3 (Contabilidad simplificada). $Y$ con respecto al sector cooperativo, lo que sugiere el CTCP es que las cooperativas que realicen la actividad de captación se ubiquen en el grupo 1 y las demás lo hagan dependiendo del tamaño de las organizaciones; sin embargo, dependerá de la autonomía administrativa de la entidad elegir el tipo de NIIF a aplicar.

\subsection{Fundamentos de la percepción empresarial}

Siendo la percepción el epicentro que permitirá conocer los criterios de los directivos abordados en esta investigación, se explora el planteamiento de Kay (2002), quien la visualiza como una herramienta de dos fases en el individuo, recepción de información y su organización en representaciones. Es válida para estudiar fenómenos que se producen al interior de las organizaciones porque 
permite comprender fenómenos culturales, hechos y patrones de conducta observables en toda empresa.

Complementariamente, para Hunt, Tourish y Hargie (2000), las percepciones de las personas son un mecanismo útil para interpretar la información, contenidos y situaciones sucedidas dentro de las organizaciones y se convierten en un poderoso elemento que influye en sus comportamientos al interior de las mismas. Las personas, a diferencia de otros seres vivos, según estos teóricos, poseen la capacidad de integrar la información que perciben y desarrollar ciertas alternativas de acción frente a las mismas; por esta razón en los estudios organizacionales, la percepción es considerada una herramienta cuyo conocimiento orienta en la toma de decisiones.

\section{Metodología de la investigación}

Teniendo en cuenta los objetivos planteados en el presente estudio, se definió el abordaje mediante el paradigma positivista, investigación de tipo descriptivo, método inductivo y un enfoque cuantitativo, orientado a determinar la percepción gerencial que tienen las empresas del sector solidario con relación a la implementación de las NIIF, para lo cual se determinó como instrumento una encuesta con un cuestionario de 7 preguntas con el cual se obtuvo información, de manera intencional, de 37 empresarios del sector objeto de estudio destacados como muestra aleatoria de la población conformada por 80 empresas solidarias en la ciudad. Los resultados del análisis se sintetizan en la Tabla 1.

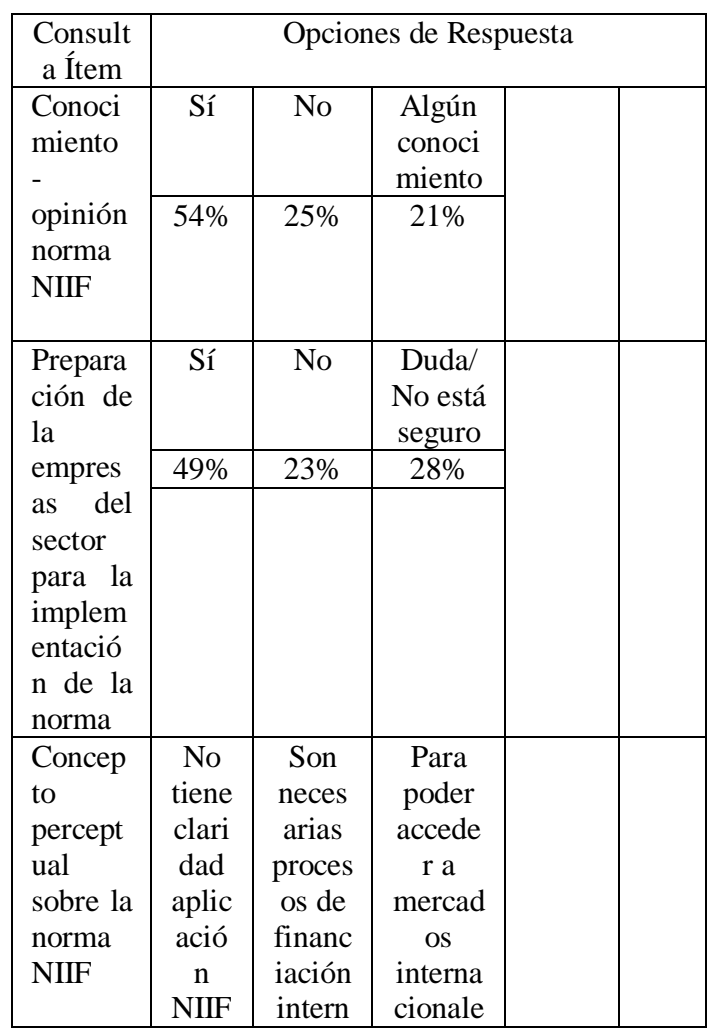

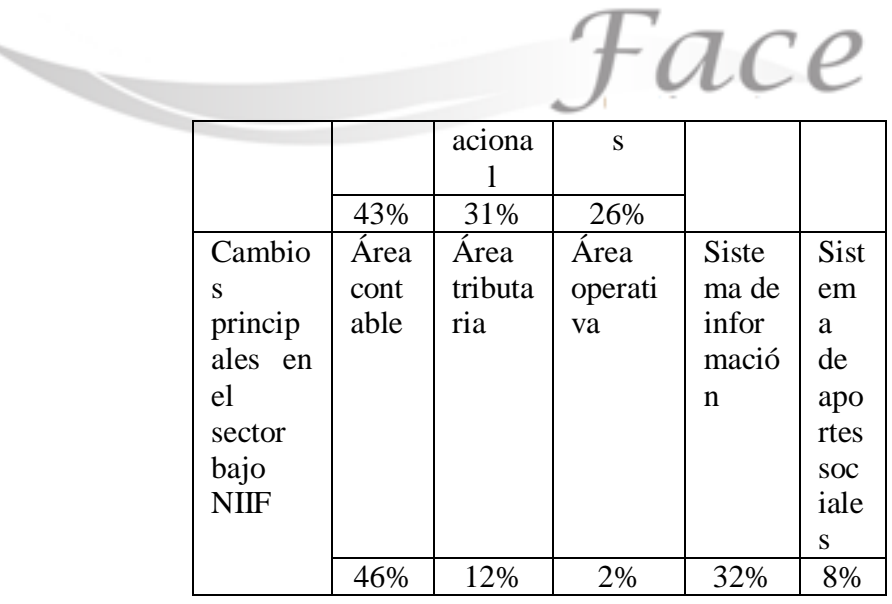

Fuente. Diseño propio. (2016). 4. Análisis de resultados y discusión

Hay un alto porcentaje de conocimiento por parte del nivel gerencial acerca de la adopción de las normas internacionales de información financiera NIIF, observándose que las que más están involucradas con este tema, son las cooperativas de mayor trayectoria y de tamaño relativamente grande, como las del sector financiero, las de construcción y agroindustria. Por el contrario, las empresas del sector más pequeñas, las que están en proceso de estructuración o no cuentan con capacidad gerencial destacada, son las clasificadas con nivel de conocimiento muy bajo o nulo respecto de las Normas NIIF. Asimismo, existe una tendencia del $49 \%$ representado por las empresas que están debidamente constituidas y con una eficiente infraestructura organizacional y financiera, en la aceptación de la implementación de las NIIF y a pensar que estas organizaciones si están preparadas para tal fin. Sin embargo, más del $50 \%$ no creen que exista un cambio sustancial en relación a la forma de llevar sus procesos contables en la actualidad; pero reconocen estar informados que sus empresas deben acogerse a este tipo de manejo de la contabilidad e información financiera. Posición de los empresarios que contrasta con las disposiciones que se vienen adelantado desde el Congreso de la República (2009) mediante leyes y la reiteración al Consejo Técnico de la Contaduría Pública para redactar las normas de convergencia, proceso que es una realidad. Su percepción sobre las NIIF (43\%) se centra en el desconcierto en cuanto a la aplicación correcta de las NIIF en el sector solidario, existiendo un efecto de mecanización para el registro de las operaciones contables, pues las entidades gubernamentales no han dado la debida difusión en cuanto a la aplicación de estas normas. Desde este sentido, se halla relación con lo postulado por Hunt, Tourish y Hargie (2000) cuando plantean que la percepción es considerada una herramienta cuyo conocimiento orienta en la toma de decisiones, pues las organizaciones aun cuando perciben la importancia, se ven limitadas por el desconocimiento de la norma. Reconocen que están orientadas a lograr una estandarización de la información contable en estas empresas y la principal causa de la 
implementación es la globalización de la economía. En segundo lugar, suman apreciaciones hacia la consideración de la significativa creciente necesidad de financiación internacional y acceso a los mercados internacionales que van adquiriendo las empresas del sector solidario a través de organismos internacionales donde se tiene en cuenta que los criterios y prácticas contables en los países se sustentan en los factores que tendrán que ser conciliados para poder implementar la armonización contable a nivel internacional, puesto que el uso de un sistema contable estandarizado, es determinante para una competencia efectiva en los mercados, no será fácil se la información no se ajusta a las NIIF, coincidiendo con lo expuesto por Luna y Muñoz (2011) al reseñar que es urgente el conocimiento y adopción de las NIIF cuya armonización de la norma está relacionada con la capacidad para vincularse apropiadamente en el nuevo mundo de los negocios, en el ambiente de economías globalizadas. Asimismo, son conscientes de los cambios sustanciales producto de la adopción de las normas internacionales, es decir, se verán afectadas varias áreas o departamentos, en especial la dependencia de contabilidad debido a que el nuevo modelo internacional presenta significativas diferencias con las leyes actuales en Colombia; también en la documentación de controles internos vinculados con el reporte financiero especialmente relacionados con procesos de cierre de estados financieros, impuestos, instrumentos financieros, propiedades, planta y equipo, y propiedades para inversión y sus correspondientes evaluaciones. $Y$ en políticas contables, en los manuales de procedimiento y revisiones a controles sobre revelaciones y procedimientos de certificación.

Producto de hallazgos y análisis efectuados se propuso como estrategia de acompañamiento un proceso de capacitación apoyado en el diseño de una cartilla con el proceso, normativa, características y condiciones del personal asesor para la implementación de las Normas Internacionales NIIF, con lenguaje técnico diseñado al alcance y comprensión de todo empresario del sector solidario de Pamplona.

\section{CONCLUSIONES:}

\section{Conclusiones}

El estudio adelantado con los representantes del sector solidario deja entrever a nivel normativo un debate en dos posturas importantes desde la percepción empresarial, por una parte es un proceso real e irreversible que parte del conocimiento de la existencia de la Ley, de la imperante adopción en el país que se evidencia en la generalidad de los empresarios del sector. Sin embargo, por otra parte se vislumbra la gran preocupación que la implementación de la Ley genera pues son más la representación de micro, pequeñas empresas del sector que las medianas 0 grandes, donde se destaca menor capacidad gerencial para entender y promover el proceso de adopción de la Ley para la implementación de las normas internacionales NIIF. Es decir, se hizo manifiesta la existencia de vacíos en el conocimiento de estas normas; así como también de inquietudes en cuanto al tema de clasificación del patrimonio de una entidad sin ánimo de lucro, como son los de las cooperativas, surgiendo interrogantes de si al diseñar el modelo fue considerado el contexto colombiano frente a los principios solidarios de este tipo de entidades.

En suma, atender los requerimientos respecto a los conocimientos, habilidades y técnicas relacionadas con normas NIIF y los principales limitantes manifestados por los gerentes como altos costos del proceso y ausencia de expertos como asesores, deben ser una prioridad inmediata para las empresas del sector solidario, no sólo para evitar sanciones sino para optar a mejores oportunidades de mercado en la economía global.

\section{REFERENCIAS:}

Berrio G. Guillermo (2015). Proceso de convergencia a NIIF en las cooperativas y sector cooperativo en Colombia. Crowe Horwath. Medellín, Colombia.

Cámara de Comercio de Pamplona (2016). Informe base de datos sector solidario. Pamplona.

Casinelli, H. (2008). La globalización del lenguaje de los negocios. Buenos Aires, Argentina: Editorial Aplicación Tributaria S.A. (p. 392).

Congreso de la República (2009). Ley 1314 de 2009. Principios y normas de contabilidad e información financiera y de aseguramiento de información. Disponible

en http://www.alcaldiabogota.gov.co/sisjur/normas/N orma1.jsp?i=36833 consultada [20/10/2016].

Consejo Técnico de la Contaduría Pública. (2012). Direccionamiento estratégico del proceso de convergencia de las normas de contabilidad e información financiera y de aseguramiento de la información, con estándares internacionales. Documento Final - CTCP

DANE (2016). Medición del Empleo Informal y Seguridad Social. Resumen Ejecutivo. Bogotá. 10 de Marzo.

Figueroa Vernor Mesén. (2008). Aplicaciones prácticas de las NIIF. Primera edición, segunda reimpresión, 314 págs.

Hunt, O., Tourish, D., \& Hargie, W. (2000). The communication experiences of education managers: Identifying strengths weakness and critical incidents. The International Journal of Educational Management, 14 (3),120-129.

Kay, M. (2002). Loving nature. Towards an ecology of emotion. London, UK: Routledge.

Luna Restrepo Juliana, Muñoz Londoño Leidy. (2011). Colombia: hacia la adopción y aplicación de las 
ISSN: 1794-9920 - ISSN-E: 2500-9338

Volumen 16 Número: 2

Año: 2016. Junio - Diciembre - Págs. 110 - 116

NIIF y su importancia. Adversia Revista virtual de estudiantes de contaduría pública. Universidad de Antioquia, No.8, pp.26-43. 